\title{
A Novel Long Term Traffic Forecast Algorithm
}

\author{
Dongchen Zhang, Shoufeng Wang, Xiaoyan Xu, Xingzheng Li, Wenwen Yao, Tinglan Wang \\ Division of Research, China Mobile Group Design Institute Co., Ltd., Beijing, China \\ A 16, Danling Street, Haidian District, Beijing, China \\ (zhangdongchen, wangshoufeng, xuxiaoyan, lixingzheng, yaowenwen, wangtinglan)@cmdi.chinamobile.com
}

\begin{abstract}
Data traffic raises dramatically in recent years. The burst of data demands for high capacity of communication networks. This brings more challenges for the industry. Forecast of traffic growth becomes important. Based on the traffic forecast, the industry can design a long-term strategy for communication development. To evaluate traffic forecast result, proper methodology is required. In this paper, a novel long term traffic forecast algorithm (LTTFA) is proposed.
\end{abstract}

traffic forecast; long-term strategy; telecommunications

\section{INTRODUCTION}

Due to the fast development of mobile traffic, spectrum resources assigned for mobile communications will become exhausted in the near future. Institutes, such as ITU, FCC, etc., have studied future spectrum requirements. Deloitte was entrusted by GSMA to check the spectrum requirement forecast in February 2011 ${ }^{[1]}$. The results proved that current networks and even the capacity expansion cannot fulfil the traffic demand in the future. Spectrum analysis shows that the Asia-Pacific region will use up all spectrum resources in 2016-2017, under the annual growth rate of $20 \%$ of the spectrum demand ${ }^{[2]}$.

As a key of the spectrum requirement forecast, traffic forecast should be taken precisely. Furthermore, long-term traffic forecast is required, since the industry needs to evaluate the amount of spectrum to meet the demands. The new spectrum application may take quite a long time, e.g., a couple of years, thus the time span for the forecast would usually be several years. However, longterm traffic forecast is rarely employed in existing researches ${ }^{[3]-[7]}$. Many studies focus on short term forecast, for example, forecasting the traffic fluctuation within a time range from tens to hundreds of hours in a limited region (such as within a cluster or cell) [3]-[6]. Only few review literature mentions the driving factors of data increase for long term forecast [7]. A prediction engine architecture in [3] forecasts the available bandwidth of the immediate future by considering indicators at lower levels of a standard communication stack. A stock price forecast method is adopted in [4] to predict short term traffic fluctuation. Theory of dynamic system reconstruction is used in [5] to forecast traffic based on historical information. The time zone of forecast method in [5] is usually hundreds of hours. [6] introduces time-series analysis for multi-vitiate data to predict rapid rise in traffic coming into the network. Method in [6] focuses on traffic rise in very short time so that the network capacity could not change. [7] reviews some driving factors, such as large screen cell phones, for long term traffic forecast. In addition, institutes in the industry published some forecasting results for different regions with hidden forecasting methods [1][2][8][15]. These forecasting results are represented and analysed in the evaluation of the case study sector in this paper.

In order to provide precise traffic growth input for spectrum requirement forecast, a Long Term Traffic Forecast Algorithm (LTTFA) is proposed in this paper. Initially, LTTFA analyses the effecting items and an interceptive S-curve for traffic forecast. Then, LTTFA studies and compares different kinds of services and terminal types. Finally, LTTFA constructs a specific forecast model in order to develop the overall traffic forecast.

The rest of the paper is organized as follows. Sector II analyses key items that affect the traffic forecast. Sector III introduces LTTFA in detail. Sector IV represents the evaluation of the case study result. Conclusions could be found in Sector VI.

\section{KEY ITEMS IN LONG-TERM TRAFFIC FORECAST}

\subsection{Population Growth}

The population is the basis of the number of users in communications networks. The number of users 
is obtained by multiplexing user penetration rate with population. The impact on the number of users of the population is reflected in the following aspects:

The natural growth of the population: the natural growth of the number of users will demand for more traffic for mobile communications.

The age composition of the population: it will affect the composition of the users of different ages. Obvious difference lies in the proportion of different ages in using the mobile communications traffic.

The education level of the population and practitioner characteristics: the level of education of the labor force has a strong correlation with its data services traffic volume and service type. The marketing strategy often specifies for different levels of education and age user. The use of multiple cell phones is popular in higher proportion of foreign workers and frequent staff between the two places.

Population urbanization: with the development of economy, urbanization gradually accelerates across the country. People are pouring into the city, and will also generate more urban mobile users.

\subsection{The development of mobile terminal}

Recently, the growth and application of the new terminal leads to high demands on the wireless network traffic. Types of mobile terminals include smart phones, netbooks, tablet PCs, e-readers, networking, game consoles and so on. These mobile terminals greatly increase the data traffic requirement. Some of these terminals act as a strong complement to traditional mobile phone, while others can completely replace the traditional mobile phone.

\subsection{New mobile application}

The new series of content-rich mobile applications is the majority of consumers and service users increasingly being sought after. Meanwhile, these new service has also led to the explosive growth of data traffic. 3G network traffic statistics developed regions in Europe and the United States, the comprehensive three main terminals (intelligent terminal, laptop, and netbook) flow analysis showed that online video applications generate 30$40 \%$ of the traffic, and will become the most important mobile data traffic growth driving factors. Ranked in second place is the web browsing, taking up $20-30 \%$ of the traffic. In addition, social networking, audio, email transceivers, software download mobile applications also become 3G data traffic sources.

\subsubsection{Online Video Applications}

Consumer demanding for online video applications trends toward rapid growth (but the price factor may be the reason for restricting this growth momentum in the future). International is widely recognized that video traffic will become the most important factor for the consumption of the wireless network bandwidth.

\subsubsection{Social Networking}

The quantity of bandwidth usage for social network is relatively low. However, the social network has significantly enhanced huge traffic amount of potential when it is combined with other applications, such as cloud storage and video. In particular, the use of mobile phones or netbooks and other mobile terminals connected social networking habits of many consumers become increasingly significant.

\subsubsection{Music flows}

Music flows are often regarded as the background service and an access to other services. Music service may lead to demand for high-bandwidth connection occupied.

\subsection{4 "Cloud" applications}

The trend of photo and video content stored in the "cloud" will encourage the terminal uplink traffic growth.

\section{LONG TERM TRAFFIC FORECAST ALGORITHM (LTTFA)}

LTTFA is proposed to forecast long term traffic volume. The result of LTTFA would present a traffic trend reference for spectrum requirement calculation. The model of LTTFA consists of the follow three parts.

\subsection{Statistical data collection of key factors}

The collection of statistical data includes development factors that affect the users of mobile services, data services development, the development of the terminal type, network construction data. For the ease of expression, key parameters are explained as follows:

Population is the basic consideration. $U_{p}$ and $r_{p}$ represent population base and population growth rate. The predicted population is expressed as $\left(1+r_{p}\right) \times U_{p}$.

Only part of the population would use mobile service. $\alpha_{u}$ is the percentage of people in proper age using cell phone, and $\gamma$ is predicting time. $\gamma$ is always valued by year. Yong people would grow up 
and start to use cell phone, thus $\left(1-\alpha_{u}\right)^{\gamma}$ of the population will be new added to mobile service subscribers.

Subscribers using different types of terminals have various data service habits. In LTTFA, percentage of each terminal type is considered. Let $l$ stands for the terminal type. $v_{l}$ refers to the percentage of terminal type $l$ in all subscribers. Thus, the number of subscribers using terminal type $l$ can be derived as:

$$
U_{l}=\left(1+r_{p}\right) \times\left(1+\left(1-\alpha_{u}\right)^{\gamma}\right) \times U_{p} \times v_{l}
$$

Based on above deriving, specific subscriber number of terminal type $l$ is calculated in Equation (1). Different kinds of services need be predicted to obtain detail traffic amount for each kind of terminals. There are many kinds of services, such as voice, short message, downloading, web browsing, social networking, etc. This paper sets $m$ as the service type. Because the capabilities vary among different types of mobile terminals, each mobile terminal type has different traffic amount for the same service. This paper defines $t_{m}^{l}$ as the traffic amount of service $m$ generated by mobile terminal type $l$. The predicted traffic for one network is presented in Equation (2).

$T=\sum_{l} \sum_{m}\left(1+r_{p}\right) \times\left(1+\left(1-\alpha_{u}\right)^{\gamma}\right) \times U_{p} \times v_{l} \times t_{m}^{l}$

\subsection{Prediction of new network technology}

New network technology usually increase data transmitting rate. This will bring better subscriber experience and increase data traffic. The adoption of new network technology is considered in LTTFA.

For network $i$, subscriber using mobile terminal type $l$ is encouraged to generate more traffic weighted by $w_{l}^{i}$. Thus the total traffic can be expressed as Equation (3).

$T_{\text {total }}=\sum_{i} \sum_{l} \sum_{m} U_{l} \times t_{m}^{l} \times\left(1+w_{l}^{i}\right)$

\subsection{Modification of total traffic using historical data}

In Equation (1) (2) (3), traffic amount is obtained based on the start point (current data) and the end point(the forecast end). Historical data for population, terminal types, service traffic amount and so on is required to predict the developing trends of each key factor.
Using linear extrapolation based on the intrinsic link between the key factors and the total traffic volume. The Byrds curve method and a linear function of the model combined the data analysis methods, is used to predict the future development of the mobile communications service situation.

Considered key factors in the traffic forecast and materials collected, the flowchart of LTTFA is shown in Fig.1.

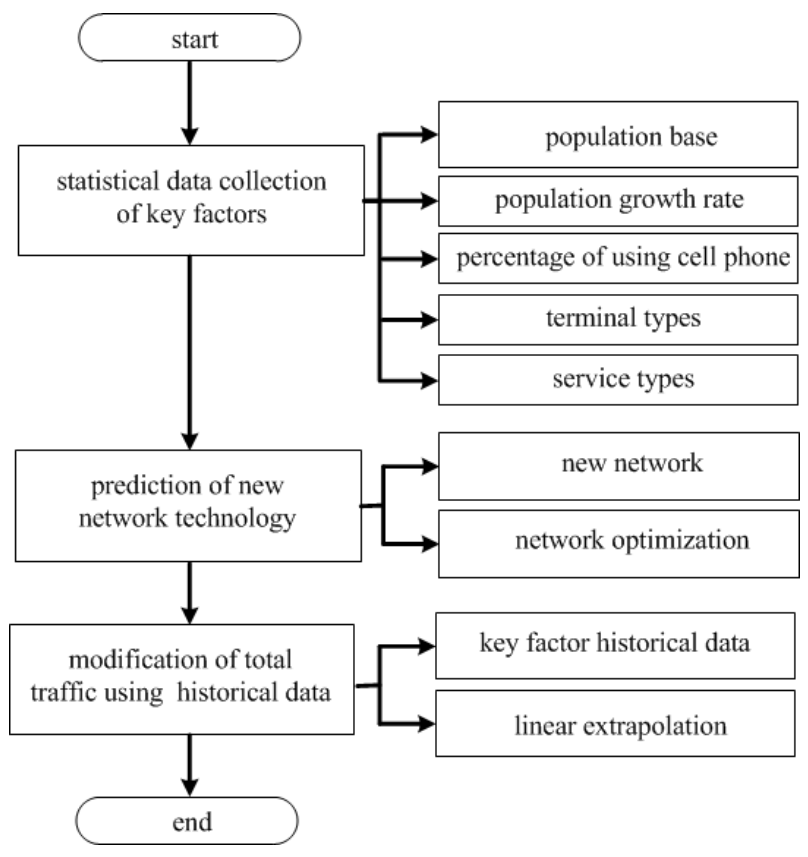

Figure 1: The flowchart of LTTFA

\section{EVALUATION OF THE CASE STUDY}

Due to the variety of reasons, only historical data in the network of China Mobile is obtained. China's total volume of data is derived on the basis of the Chinese mobile traffic data translated in accordance with the number of mobile users in China and the proportion of national users.

\subsection{Voice Traffic Forecast}

Voice traffic forecast considering the amount of users and the growth rate of the user MOU and the growth rate of the billing duration. The total billing duration is the cumulative of length per user billing.

The voice traffic forecast result for China is depicted in Fig. 2. 


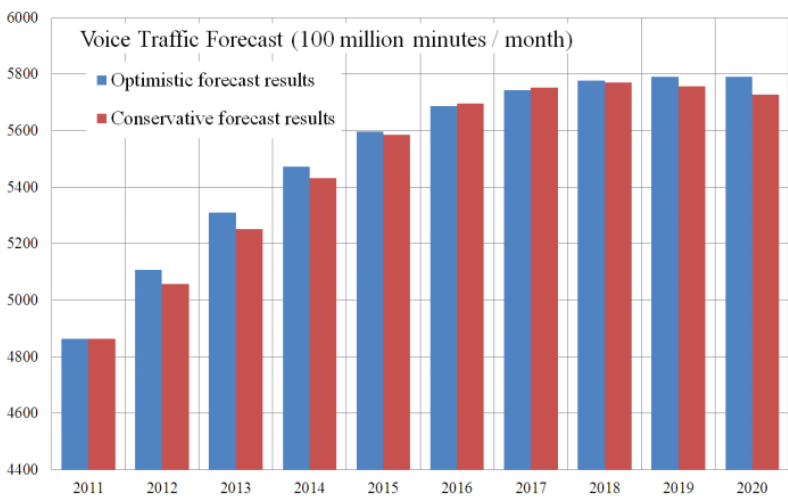

Figure 2: Voice traffic forecast for China

\subsection{Data Traffic Forecast}

2012-2020 growth rate forecast for data traffic is primarily based on the 2010 and 2011 survey data. Changes in the long-term growth rate is generally expressed as a four morphology that grows linearly concave function, convex function and S-curve key parameter prediction, the empirical method can be used to select the optimal prediction results. Forecasting market trends as shown in Fig. 3 and Fig. 4.

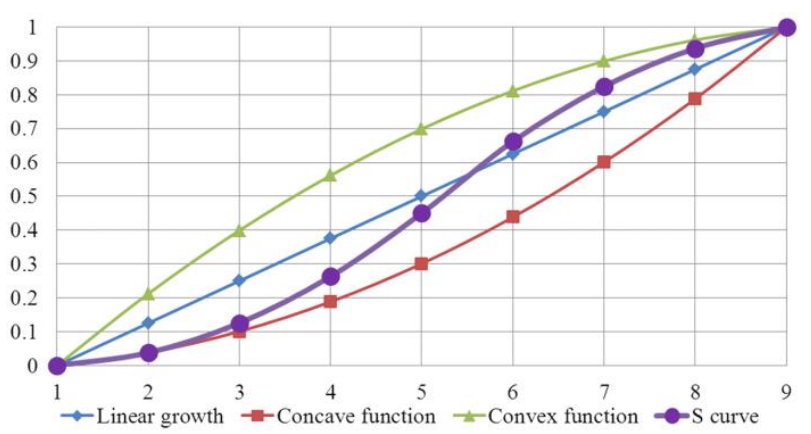

Figure 3: Growth trend line

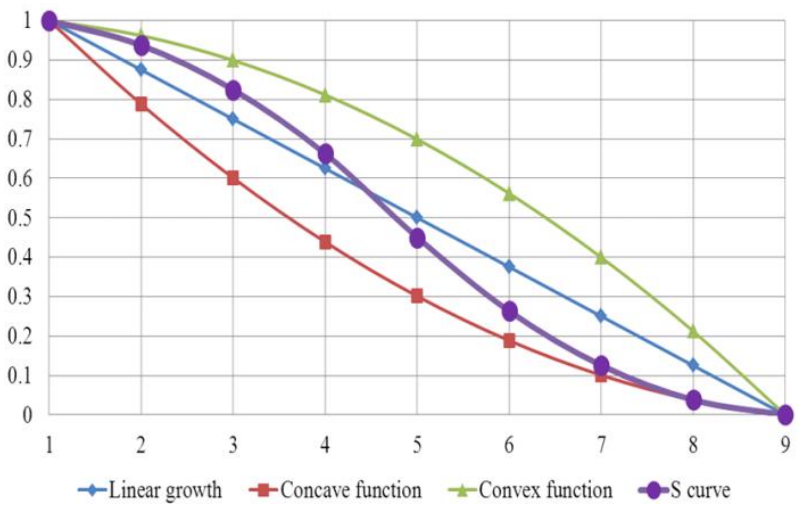

Figure 4: Reduce trend line

Based on the above multifaceted impact factors, the final total data traffic forecast results is shown in Fig. 5 and Fig. 6. From Fig. 5 and Fig. 6, the limit of spectrum resources and other factors on the development of the traffic volume is represented. Especially in the late predict midterm, the optimistic forecast results increase more quickly than the results of the conservative forecast.

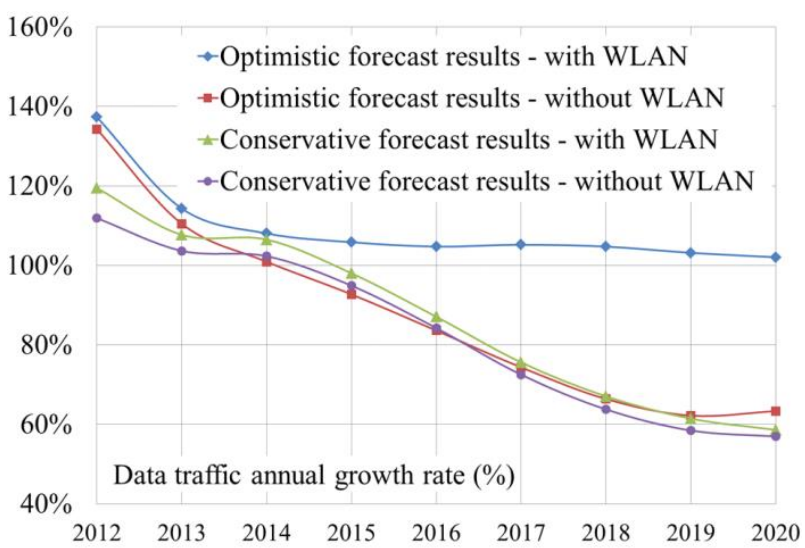

Figure 5: Data traffic annual growth rate

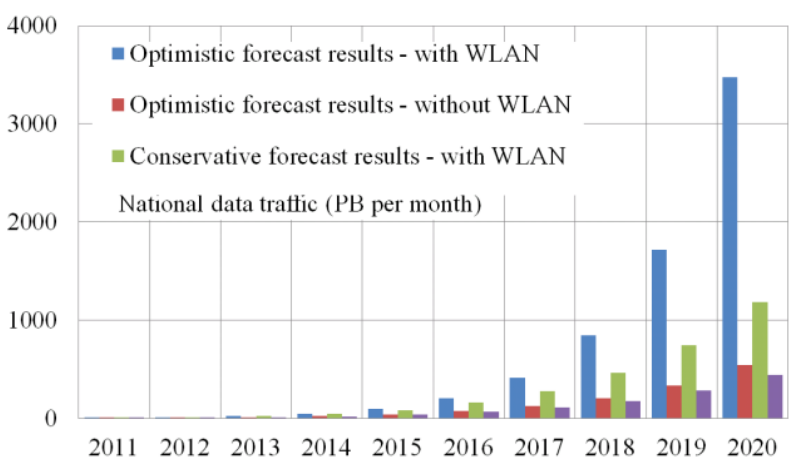

Figure 6: National data traffic

\section{CONCLUSIONS}

As data traffic keeps rising in recent years, the allocated spectrum will run out soon. Long term traffic forecast is employed for deciding new spectrum for telecommunications. Existing researches focus on short term traffic forecast, but few studies mention long term forecast. This paper proposes a Long Term Traffic Forecast Algorithm (LTTFA) to give a solution to long term traffic forecast. Based on the comprehensive analysis of the international traffic forecasts and the actual situation in China, LTTFA defines a set of key factors in traffic forecast, and presents a traffic forecast for China between 2012 to 2020.

\section{ACKNOWLEDGEMENT}

This work was supported in part by National Major Science \& Technology Specific Projects-Next Generation Broadband Wireless \& Mobile Communications (2013ZX03003016 and 2012ZX03006003-005).

\section{REFERENCES}

[1]. Deloitte Spectrum Study (2011) Meeting mobile demand: an assessment of future 
mobile spectrum requirements. America: GSMA..

[2]. CJK-IMT White paper No 03 (2012) Forecast of mobile broadband development in the Asia Pacific Region. CJK-IMT WG: Geneva, Switzerland: international Telecommunications Union.

[3]. Hentschel, Uwe, Schmidt, Alexander.(2011). Predictable Communication for Mobile Systems. In: 2011 14th IEEE International Symposium on Object/Component/ServiceOriented Real-Time Distributed Computing, America: IEEE. 24-28.

[4]. Xiaodong Wang, Ximing Hu. (2012). Mobile traffic forecasting based on the relative position of multi-scale average lines. In: 2012 International Conference on Information Science and Technology, America: IEEE. 279283.

[5]. Jianmin Wang, Yu Peng, Xiyuan Peng (2009). Mobile communication traffic forecast based on a new fuzzy model. In: Instrumentation and Measurement Technology Conference. America: IEEE. 872-877.

[6]. Yoshikazu Akinaga, Shigeru Kaneda, Noriteru Shinagawa, Akira Miura (2005). A proposal for a mobile communication traffic forecasting method using time-series analysis for multivariate data. In: IEEE Global Telecommunications Conference. America: IEEE. 29-33.

[7]. Stordahl, K., Elnegaard, N.K (2012). Longterm mobile data traffic forecasts for the Western European market. In: Telecommunications Network Strategy and Planning Symposium, America: IEEE. 1-6.

[8]. UMTS Forum Report No 44 (2011). Global mobile network traffic - a summary of recent trends. America: UMTS Forum.

[9]. CCSA TC6 report No 95 (2011). The next ten years the IMT the business market forecast. Beijing, China: CCSA.

[10]. APG-5 OUT-03 (2011). Information of White Paper on Forecast of mobile broadband development in the Asia-Pacific Region. Thailand: APG.

[11]. OBI technical paper No 33 (2010). Mobile broadband: the benefits of additional spectrum. America: Federal Communications Commission.

[12]. 4G America white paper No 483 (2011). Slides of Mobile Broadband Explosion: 3GPP broadband evolution to IMT-Advanced. America: 4G America.
[13]. R07-WP5D-GSMA (2011). Global mobile network traffic-looking at mobile traffic growth since the year 2007 towards revision of report ITU-R. England: GSMA.

[14]. Ericsson report No 97. Traffic and market data report on the pulse of the networked society. Swaziland: Ericsson.

[15]. Information Centre report 339(2011). 29th China Internet Development Statistics Report China Internet Network. .Beijing China: MIT. 